\title{
La importancia de Pelagra en el diagnóstico diferencial
}

\author{
The importance of pellagra in the differential
}

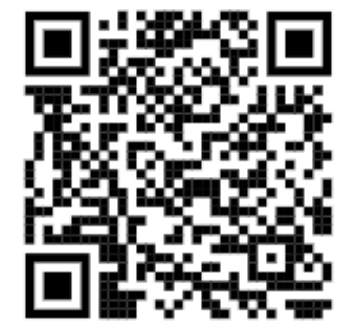

\author{
${ }^{1}$ Dr. Sebastián Rojas Chaves \\ Clínica Dr. Marcial Fallas Díaz, San José, Costa Rica \\ drtansebas@gmail.com \\ https://orcid.org/0000-0002-1810-3388 \\ ${ }^{2}$ Dra. María Fabiola Cárdenas Quirós \\ Emergencias Médicas Monteverde, San José, Costa Rica \\ dramfcq@gmail.com \\ https://orcid.org/0000-0002-9126-2553
}

RECIBIDO

CORREGIDO

ACEPTADO

$28 / 02 / 2019$

$15 / 03 / 2019$

$26 / 03 / 2019$

\section{RESUMEN}

Pelagra es el nombre que se le ha dado a la condición poco conocida, resultado del déficit de vitamina B3 o niacina, una enfermedad potencialmente mortal la cual es caracterizada por una triada clásica de diarrea, dermatitis y delirio, que está asociada a factores de riesgo como desnutrición, pobreza, ingesta de ciertos medicamentos, alteraciones metabólicas entre otros. Pelagra es una enfermedad de diagnóstico clínico que difícilmente se considera en los diagnósticos diferenciales de pacientes que consultan en servicios de emergencias o que se encuentran internados en salones de hospitales y es de suma importancia debido a la mortalidad que conlleva un diagnóstico tardío.

PALABRAS CLAVE: niacina; pelagra; avitaminosis; dermatitis; alcoholismo.

\section{ABSTRACT}

Pellagra is the name of a condition resulting from the vitamin B3 or Niacin deficiency, a potential deathly desease which is characterized by a classic triad of Diarrhea, Dermatitis, and Delirium, which is associated with risk factors such as malnutrition, poverty, intake of certain drugs, metabolic disturbances, among others. Pellagra's diagnosis can be made on clinical grounds alone, but it's hardly in the clinicians differential for hospitalized patients or in the emergency department; this is of great importance due the mortality that represents a late diagnosis.

KEYWORDS: niacin; pellagra; avitaminosis; dermatitis; alcoholism
${ }^{1}$ Médico general, graduado de la Universidad Autónoma de Centroamérica (UACA). Código médico: 13837

drtansebas@gmail.com

${ }^{2}$ Médico general, graduada de la Universidad Autónoma de Centroamérica (UACA). Código médico: 14496 dramfcq@gmail.com 


\section{INTRODUCCIÓN}

Pelagra fue descrita por primera vez por Don Gaspar Casal en 1735 (1) y fue nombrada por Frapolli en 1771(2) debido a sus manifestaciones dermatológicas; proviniendo el nombre del italiano "Pela", significando piel, y "agra", significando áspera (3); pero no fue hasta 1926 cuando Goldberger identifica la nicotinamida, una forma de B3, como el factor preventivo de pelagra (4). Esta condición desde sus inicios se ha relacionado con desnutrición y pobreza (1), así como con alcoholismo $(1,5)$ y en años más recientes se ha relacionado también a la ingesta de ciertos medicamentos, condiciones de malabsorción, entre otros (6). Gracias al enriquecimiento de alimentos con niacina, la pelagra ha sido casi erradicada de la población general, (7) lo que puede llevar a que muchos clínicos no incluyan pelagra en diagnóstico diferencial incluso de pacientes con desnutrición (8).

\section{MATERIALES Y MÉTODOS}

Se ha realizado una revisión sistemática de documentos científicos de índole médico, así como guías, reportes de casos clínicos y libros de nutrición, utilizando motores de búsqueda como Pubmed y Elsevier. Se seleccionaron las fuentes que presentaran información tanto de aspectos fisiopatológicos como cuadro y manejo clínico. Se excluyeron un artículo cuyo enfoque era meramente bioquímico, dos artículos que constaban solamente de imágenes y 6 artículos que se enfocaban en múltiples tipos de avitaminosis debido a que la mención de la pelagra era mínima.

\section{EPIDEMIOLOGÍA}

FA diferencia del pasado cuando se presentaban epidemias de pelagra por déficit de consumo de vitamina B3 en las poblaciones más pobres, sobre todo aquellos que tenían una dieta primariamente de trigo y cereales (7), en la actualidad la incidencia de pelagra es muy rara debido a un aumento en la vigilancia y estrategias tales como enriquecimiento vitamínico de alimentos (9) por lo que se presentan solamente casos esporádicos los cuales ocurren en alcohólicos crónicos, personas que siguen "dietas de moda", o personas dependientes de drogas y pacientes en estados de malabsorción (3).

\section{FISIOPATOLOGÍA}

Para hablar de la fisiopatología de la pelagra, primero hay que entender el rol en el metabolismo de la Niacina, una sustancia soluble en agua, que no tiene almacenamientos apreciables en el cuerpo (8). La niacina funciona como una coenzima o cosubstrato en una amplia cantidad de reacciones de reducción y oxidación o Redox en la forma de Nicotinamida adenina dinucleótido hidrógeno (NADH) y Nicotinamida adenina dinucleótido fosfato hidrógeno (NADPH) (10). En mamíferos, la nicotinamida es biosintetizada desde triptófano (Trp) (11). Un miligramo de niacina es igual a un equivalente de niacina (NE); el cual se encuentra naturalmente en carnes como nicotinamida y en plantas como acido nicotínico (12), $1 \mathrm{mg}$ de cada uno de estos equivale a $1 \mathrm{NE}$ (13). Si no se consume suficiente niacina, se puede convertir el 
$60 \mathrm{mg}$ de triptófano en 1 NE (12), sin embargo esta conversión requiere cantidades adecuadas de tiamina (vitamina B1), Riboflavina (vitamina B2), Piridoxina (Vitamina B6) y NADP. (14). La cantidad necesaria de NE diaria dependerá de género y estado de gravidez, requiriendo hombres $16 \mathrm{NE}$, mujeres no embarazadas 14NE $y$ embarazadas 18NE (13).

Al ser la niacina necesaria para una adecuada función celular por su papel en la glucolisis, metabolismo de proteínasaminoácidos, metabolismo del piruvato, biosíntesis de la pentosa, generación de uniones de alta energía, y metabolismo de ácidos grasos; múltiples órganos y tejidos se afectan por el déficit de NE, causando expresiones clínicas diversas.

\section{ETIOLOGÍA}

Como se mencionó previamente, la pelagra es causada por un déficit tanto de niacina como de su precursos el triptófano, y otras vitaminas del complejo $B$; este déficit puede darse debido a múltiples causas: 1-Alcoholismo, 2- déficit de ingesta: déficit dietético, anorexia nerviosa, nutrición parenteral total, 3Estados de mala absorción: enfermedad de Crohn, colitis ulcerativa, enfermedad celiaca, carcinoma esofágico, síndrome de malabsorción pediátrica, amiloidosis, complicaciones asociadas a cirugía gastrointestinal, 4-perdida excesiva: hemodiálisis, 5-alteraciones metabólicas: Enfermedad de Hartnup (defecto en el mecanismo de transporte de triptófano), síndrome carcinoide, 5- Pelagra inducida por drogas como: Isoniazida, pirazinamida, etionamida, azatioprina, 5fluoracilo, quimioterapia, valproato de sodio, glibenclamida entre otros (6).

\section{MANIFESTACIONES CLÍNICAS}

La pelagra ha sido clásicamente descrita con una tríada conocida como "las 3 Ds" dermatitis, diarrea y demencia, agregando "Death/muerte" como cuarta D si no se trata (15). Es importante recalcar que generalmente no se observa la triada completa en estos pacientes, y a pesar que es llamada Pelagra por el hallazgo dermatológico, este puede no estar presente (16-18); otro dato importante es que aunque se ha referido la demencia como parte de la tríada de la pelagra, la alteración neurocognitiva que se presenta es mejor caracterizada como delirio e incluso este puede ser el único síntoma de la enfermedad (8).

\section{- Piel}

La dermatitis causada por pelagra es bilateral y simétrica, presentándose en áreas de exposición solar $(4,19)$. Inicia como un eritema agudo o intermitente que cambia gradualmente a una erupción exudativa en el dorso de las manos, cara, cuello y tórax con sensación de prurito y dolor al tacto. Un hallazgo característico es una erupción bien marcada en el cuello llamado "Collar de Casal" el cual cubre el cuello completamente en el área de los dermatomas C3 y C4 $(3,6,8)$. Eventualmente los pacientes muestran una piel engrosada que es seca, agrietada e hiperqueratósica con apariencia similar a un pergamino $(3,6,8)$.

\section{- Gastrointestinal}

Los síntomas gastrointestinales se piensa que son debidos a una inflamación generalizada del canal alimenticio, se puede presentar como estomatitis, 
glositis, nausea, vomito, constipación, dolor abdominal y por último diarrea intratable (20).

\section{- Neurológico}

Como se mencionó previamente, la alteración neurocognitiva asociada a pelagra es más consistente con Delirio que con demencia (8), el paciente puede presentar distintas manifestaciones que incluyen cefalea, irritabilidad, ansiedad, alucinaciones, estupor, apatía, fotofobia, tremor, ataxia, paresia espástica, fatiga y depresión $(3,4)$. Puede progresar a una encefalopatía en casos severos, que es caracterizado por confusión, psicosis, desorientación, delirio y por último un estado comatoso y finalmente la muerte $(8,21)$.

\section{DIAGNÓSTICO}

El diagnóstico de pelagra puede realizarse basado en clínica (22), utilizando la triada clásica de diarrea, dermatitis y delirio, en pacientes con historia de malnutrición o alcoholismo, a pesar que la aparición de la triada completa suele ser poco frecuente.

El uso de exámenes no es necesario, medir niveles de Niacina (bajo $<0.5 \mu \mathrm{g} / \mathrm{dl}$, normal 0.5 a $8.45 \mu \mathrm{g} / \mathrm{dl}$ ) (23) o metabolitos urinarios de niacina son técnicas de laboratorio disponibles que ayudan a confirmar el diagnóstico.

A las 48 horas de iniciar tratamiento los síntomas gastrointestinales y dermatológicos generalmente resuelven, por lo tanto el diagnostico se confirma con la resolución rápida de los síntomas (24). La respuesta al tratamiento de los síntomas neurocognitivos es más variable, pudiendo resolver en pocos días, hasta presentar déficit permanente (8).

\section{TRATAMIENTO}

El tratamiento recomendado por la organización mundial de la salud para adultos con pelagra es nicotinamida $100 \mathrm{mg}$ vía oral tres veces al día o 50mg cada doce horas vía parenteral y el tratamiento se debe continuar por 3-4 semanas. Además que se recomienda administrar preparaciones con complejo de vitaminas $B$ porque estos pacientes generalmente presentan déficit de otras vitaminas del complejo B (24).

\section{CONCLUSIÓN}

En la actualidad gracias a esfuerzos de distintas organizaciones mundiales $y$ locales, se ha logrado disminuir considerablemente la incidencia de enfermedades relacionadas a déficits nutricionales, sin embargo aunque en menor frecuencia estas aún se presentan en poblaciones de riesgo y es necesario saber reconocerlas.

La pelagra es una enfermedad poco frecuente, que generalmente no se encuentra en nuestros diagnósticos diferenciales y debemos considerarla en todo paciente con signos de desnutrición o historia de alcoholismo. Es importante que si se tiene la sospecha de que un paciente presenta esta condición se debe iniciar tratamiento sin esperar el resultado de los exámenes de laboratorio ya que el diagnóstico es principalmente clínico. 


\section{REFERENCIAS}

1. Wilson WH. The Diet Factor in Pellagra. Journal of Hygiene. 1921 07;20(01):1-59. https://doi.org/10.1017/s0022172400043205

2. Blue R. Pellagra in California. California state journal of medicine. 1910; 8(3): 101-102

3. Hegyi J, Schwartz RA, Hegyi V. Pellagra: Dermatitis, dementia, and diarrhea. International Journal of Dermatology. 2004 01;43(1):1-5. https://doi.org/10.1111/i.1365-4632.2004.01959.x

4. Hegyi V. Dermatologic Manifestations of Pellagra. Medscape [internet]. 2018 Feb 206 [cited 2019 Jan 15]. Avariable from: https://emedicine.medscape.com/article/1095845-overview

5. Klauder JV, Winkelman NW. Pellagra among chronic alcoholic addicts. Journal of the American Medical Association. 192802 04;90(5):364-371. https://doi.org/10.1001/jama.1928.02690320026008

6. Li R, Yu K, Wang Q, Wang L, Mao J, Qian J. Pellagra Secondary to Medication and Alcoholism. Nutrition in Clinical Practice. 201608 19;31(6):785-789. https://doi.org/10.1177/0884533616660991

7. Park YK, Sempos CT, Barton CN, Vanderveen JE, Yetley EA. Effectiveness of food fortification in the United States: the case of pellagra. American Journal of Public Health. 2000 05;90(5):727-738. https://doi.org/10.2105/ajph.90.5.727

8. Oldham MA, Ivkovic A. Pellagrous encephalopathy presenting as alcohol withdrawal delirium: A case series and literature review. Addiction Science \& Clinical Practice. 201207 06;7(1). https://doi.org/10.1186/1940$\underline{0640-7-12}$

9. Matapandeu G, Dunn SH, Pagels P. An Outbreak of Pellagra in the Kasese Catchment Area, Dowa, Malawi. The American Journal of Tropical Medicine and Hygiene. 201702 20; 96(5): 1244-1247. https://doi.org/10.4269/ajtmh.16-0423

10. Sardesai V. Introduction to Clinical Nutrition. 2 ed. New York: CRC Press; 2003. 179-186 p.

11. Terakata M, Fukuwatari T, Kadota E, Sano M, Kanai M, Nakamura T, Funakoshi H, Shibata K. The Niacin Required for Optimum Growth Can Be Synthesized from L-Tryptophan in Growing Mice Lacking Tryptophan-2,3-Dioxygenase. The Journal of Nutrition. 201305 22;143(7):1046-1051. https://doi.org/10.3945/in.113.176875

12. Wildman RE, Medeiros DM. Advanced Human Nutrition. Florida: CRC Press; 2000. 521p.

13. Institute of Medicine (US) Standing Committee on the Scientific Evaluation of Dietary Reference Intakes and its Panel on Folate, Other B Vitamins, and Choline. Dietary Reference Intakes for Thiamin, Riboflavin, Niacin, Vitamin B6, Folate, Vitamin B12, Pantothenic Acid, Biotin, and Choline. Washington (DC): National Academies Press (US); 1998. 123-149 p.

14. Dalgliesh CE. Interrelationships of tryptophan, nicotinic acid and other b vitamins. British Medical Bulletin. 1956 01;12(1):49-51. https://doi.org/10.1093/oxfordjournals.bmb.a069514

15. World Health Organization. The management of nutrition in major emergencies. Geneva: WHO Library Cataloguing-in-Publication; 2000. 31-32p.

16. Dawson B, Favaloro EJ, Taylor J, Aggarwal A. Unrecognized pellagra masquerading as odynophagia. Internal Medicine Journal. 2006 07;36(7):472-474. https://doi.org/10.1111/j.1445-5994.2006.01108.x

17. Kertesz SG. Pellagra in 2 Homeless Men. Mayo Clinic Proceedings. 2001 03;76(3):315-318. https://doi.org/10.4065/76.3.315

18. Spivak JL, Jackson DL. Pellagra. An analysis of 18 patients and a review of the literature. In Johns Hopkins Medical Journal. 01 Jun 1977; 140(6):295-309.

19. Karthikeyan K, Thappa DM. Pellagra and skin. International Journal of Dermatology. 2002 08;41(8):476481. https://doi.org/10.1046/i.1365-4362.2002.01551.x 
20. Junqueira-Franco MVM, Troncon E, Garcia P, Del Alma M, Jordao A, Vannucchi H. Intestinal permeability and oxidative stress in patients with alcoholic pellagra. Clinical Nutrition. 2006 Dec;25(6):977-983. https://doi.org/10.1016/i.clnu.2006.03.010

21. Charness ME. Brain Lesions in Alcoholics. Alcoholism: Clinical and Experimental Research. 1993 02;17(1):2-11. https://doi.org/10.1111/i.1530-0277.1993.tb00718.x

22. Brown TM. Pellagra: An Old Enemy of Timeless Importance. Psychosomatics. 2010 03;51(2):93-97. https://doi.org/10.1016/s0033-3182(10)70668-x

23. Cambridge Biomedical.Vitamin B3 Niacin in Plasma [internet]. Cambridge: Cambridge Biomedical Inc. 2016 [cited: 2019 feb 20]. Avariable from: https://www.cambridgebiomedical.com/s/Vitamin-B3.pdf. Accessed $\underline{2016 \text { May } 05}$

24. World Health Organization. Pellagra and its prevention and control in major emergencies. USA: World Health Organization. 2000 [cited: 2019 feb 20]. Avariable from: from https://www.who.int/nutrition/publications/en/pellagra prevention control.pdf 